\title{
THE OPHTHALMOSCOPICAL APPEARANCE OF THE FUNDUS OCULI IN ELDERLY PERSONS WITH ARTERIO-SCLEROSIS AND NORMAL BLOOD PRESSURES*
}

\author{
BY
}

\author{
H. Vogelius AND P. BechGaARD
}

From the Medical Department II (Dr. H. Heckscher), and the Ophthalmological Department (Prof. E. Holm), Municipai Hospital, Copenhagen.

IN considering the pathological changes in the retinal vessels it is important to know which changes are due to arterio-sclerosis and which to hypertension. On this question there is, however, considerable uncertainty.

Berglund (1945) has reviewed the literature and criticizes the usual accepted classification.

Kornerup (1947), who gives an excellent review of the literature, assumes purely hypertensive changes to be present when the arteries show increased fullness and an exaggerated reflex streak and slight vestiges of crossing phenomena, "indentations ". The localized and general narrowings of the arteries have been assumed to be of spastic origin, if they are reversible, and if the vessels in the narrowed parts have normal transparency and no other signs of thickening of the walls. He assumes arterio-sclerosis to be present when the calibre of the artery is distinctly and irreversibly reduced, locally or generally, especially when the arteries have lost their transparency. 111 kinds of well-marked crossingphenomena, especially those where the artery is opaque, further, tortuosity of the perimacular vessels and sheathing of the vessels of the 2 nd and 3rd order, have each one separately and still more all together been assumed to indicate arteriosclerosis.

Elwyn (1944) regards the following appearances as characteristic of hypertensive retinopathy :

(1) Oedema of retina and papilla.

(2) "Cotton wool exudates".

(3) Haemorrhages.

(4) Sharply defined white spots in the deeper layers of the retina.

(5) Stellate macula.

Wagener (1939) regards the following as characteristic of arterio-sclerosis :

(1) Even constriction of the vascular lumen, without changes in calibre.

(2) Alteration of the vascular reflex.

(3) Arterio-venous compression.

(4) Localized spasms-irregularities of calibre.

(5) Peri-arterial sheathing.

$$
\text { * Received for publication October 1, } 1949 .
$$


Gans (1944) says that hypertensive and arterio-sclerotic changes are usually present together, and uses the following classification (A.: arterio-sclerosis; $\mathrm{H}$ : hypertension) :

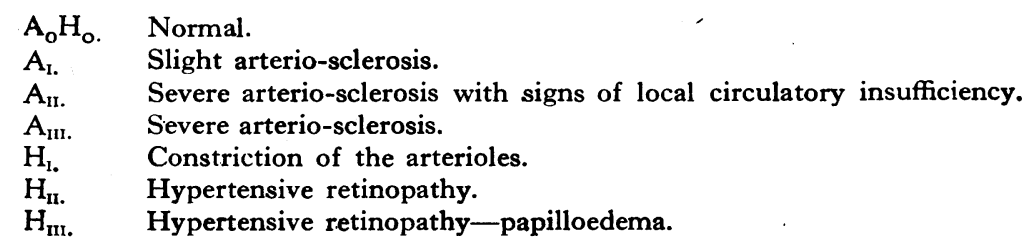

The classifications of Gans and Wagener differ in that Gans regards the constrictions of the arterioles (spasms) as hypertensive, and Wagener regards them as arterio-sclerotic. Gans does not point out which signs he believes to be characteristic of arteriosclerosis and which of hypertension.

Woods and Peet (1941) have classified cases of hypertensive retinopathy according to their severity; in this grouping the significance of spasms is stressed :

Group 1. Normal ophthalmoscopic appearance.

Group 2. Arterio-sçlerosis without spasms.

Group 3. Definite spasms with or without irregularities of calibre, with or without haemorrhages or exudates.

The classification of retinal changes in hypertension which is most used at the present time is that suggested by Wagener and Keith (1937) :

Group 1. Slight constriction of the arteries or slight arterio-sclerosis.

Group 2. More marked constriction of the arteries or more marked arteriosclerosis.

Group 3. The same plus exudates and haemorrhages.

Group 4. The same plus papilloedema.

The classifications of Woods and Peet, and of Wagener and Keith, do not deal with the differential diagnosis between hypertensive and arterio-sclerotic changes.

When we were studying the retinal changes in hypertension we could find in the literature no account of the appearance of the retina in the older age-groups based on a large group of persons with normal blood pressures. As such an account seems essential if one is to distinguish between hypertensive and arterio-sclerotic changes, the appearance of the retina of elderly persons with normal blood pressures is described here.

\section{Material}

124 subjects (85 men and 39 women) with normal blood pressures, as confirmed by repeated recordings, have been examined. The blood pressures were below $140 \mathrm{~mm}$. Hg. systolic and $90 \mathrm{~mm}$. $\mathrm{Hg}$. diastolic. The ages varied from 40 to 95 years, 25 in each 10-year group, except in the last in which there were only three persons over 90 years of age. The subjects over the age of 65 years were chosen from the old people's home; all of them had clinical signs of arterio- 
sclerosis. Those under 65 years were either normal subjects or patients from the Copenhagen Municipal Hospital suffering from conditions not affecting the vascular system.

\section{Method}

The ophthalmoscopic examinations were all made by one ophthalmologist; mydriasis was used in all cases.

Particular attention was paid to the following: arterial diameter (including irregularities of calibre and spasms), arterio-venous crossings, course and reflex of the arteries.

The condition of the veins and retina was also studied, the latter with special attention to haemorrhages, exudates, thromboses, and the papilla.

The results appear in the Table opposite.

\section{Results}

Arteries.-Changes probably due to arterio-sclerosis (abnormal arterio-venous crossings, the veins being slightly depressed, raised, or displaced sideways) were seen even in the youngest age-group (40-50 years). Slight irregularities of calibre were also seen in this group, but constriction of the arteries was seen in only one case under 60 years. The frequency of abnormal arterial diameters (narrowed or irregular) and of abnormal arterio-venous crossings increases rapidly at 60 years and continues to increase with age. Irregularities of calibre were found in all the 49 cases of over 70 years of age. The frequency of abnormal reflex also increases with age.

In only three out of the 124 cases was the course of the arteries abnormally tortuous. It must however be emphasized that the opinion whether an artery is tortuous or not is purely subjective, and cannot be assessed objectively like arterial constriction or irregularities of calibre.

Compression of the vein with peripheral dilatation and tapering towards the artery was seen in only two cases. Spasms (temporary or permanent marked constrictions of varying lengths along an otherwise normal artery) were not seen.

Veins.-Nothing notable. It may be mentioned that no thromboses were seen.

Retina.-Haemorrhages, exudates, blurred disk edges, or papilloedema were not seen. In the older age groups some pale disks and various changes in the macula were recorded, but they were not associated with any decrease in visual acuity. Only five of the 124 subjects had a visual acuity below 6/18 with proper correction.

\section{Summary}

The fundus oculi of 124 persons over 40 years of age with normal blood pressures, and in many cases with marked arterio-sclerosis, have been examined ophthalmoscopically. Changes in the retinal 
The Fundús Oculi and Arterio-Sclerosis

TABLE

Findings in the Fundus Oculi of 124 Elderly Persons with Normal Blood Pressures

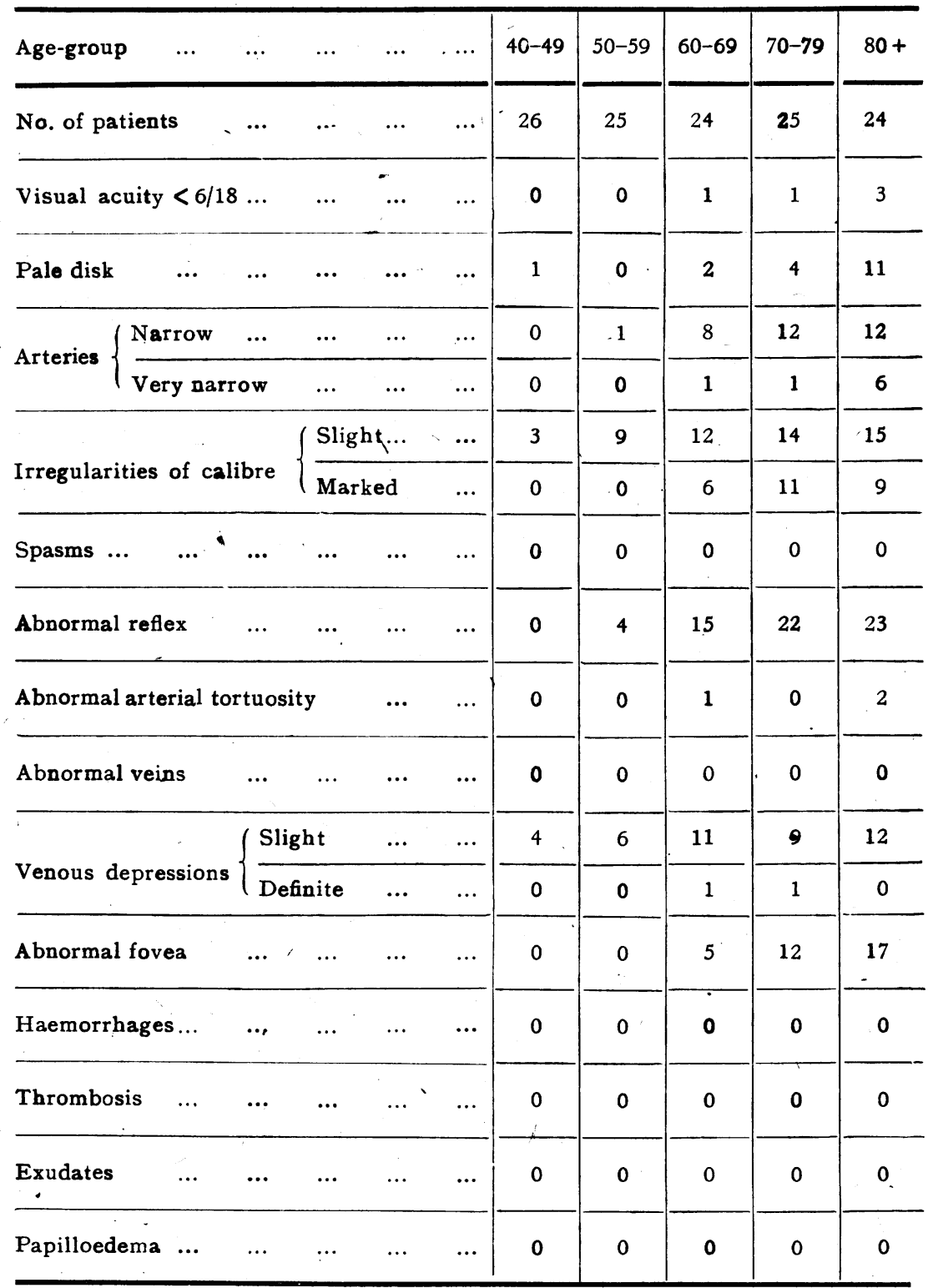


arteries (slight irregularities of calibre, and slight changes at the arterio-venous crossings) were found in a few cases in the 40-50 years age group. Over the age of 60 years these changes increased in degree and frequency, and many constricted arteries were found.

These two types of change correspond with the alterations occurring in patients with hypertension in Wagener and Keith's Groups 1 and 2.

Spasms of the arteries and retinopathy were not seen.

We should like to express our thanks to chief physician T. Geill, M.I. for permission to examine his patients.

\section{REFERENCES}

Berglund, H. (1945). Nord. Med., 27, 1809.

Elwy., H. (1944). Arch. Ophthal., Chicaso, 31, 376.

GANS, J. A. (1944). Ibid., 32, 267.

Kornerup, T. (1947). Acta ophthal. Kbh., Suppl. 28.

WAGENER, H. P. (1939). Trans. Amer. Acad. Ophthal, p. 54.

and KelTH, N. M. (1937). XV Concil. Ophthal., 1, 1.

Woods, W. W., and Plet, M. M. (1941). J. Amer. med. Assoc., 117, 1508.

\section{BOOK NOTICE}

Amsler Charts. Published by Hamblin, London, 1950. Pp. 12, with the 7 charts in Loose-leaf bock. Price 3\%/6.

Most British ophthalmologists will be familiar with the methods of qualitative analysis of the central visual field elaborated and introduced by Professor Marc Amsler of $Z$ ürich. The main chart of Amsler's system consists of a grid of 400 squares ruled in white on a black background, with a white fixation spot in the centre; the grid is so arranged that when held $28-30 \mathrm{~cm}$. from the eye, the sides of the whole pattern of squares subtend angles of $20^{\circ}$, and each small square an angle of $1^{\circ}$ at the nodal point. The network therefore, on the retina, would occupy, when the central point is fixed, an area of $10^{\circ}$ all round the fixation point, thus covering the whole macular area but not extending out temporally as far as the blind spot. This chart with various modifications of the primary pattern affords a rapid, and apparently accurate, method of analysing subjectively the visual disturbances which accompany the beginning and evolution of macular disturbances. These are mapped out by the patient as areas of disappearance, distortion, etc., of the grid pattern, findings which can be drawn on the duplicate pad of charts supplied in the book.

The method, of which we have some experience, is of considerable value in routine clinical work, and ophthalmologists should welcome the opportunity of obtaining the charts, with full and explicit instructions for their use, in a convenient loose-leaf form. 